\title{
TTR
}

Traduction, terminologie, re?daction

\section{Dupuis, Hector, Romain Légaré et Michel Therrien, Dictionnaire des synonymes et des antonymes, Éditions Fides, Montréal, 2003, 720p.}

\section{Chantal Bouchard}

Volume 16, numéro 1, 1er semestre 2003

Controverse en traductologie

Controversy in Translation Studies

URI : https://id.erudit.org/iderudit/008567ar

DOI : https://doi.org/10.7202/008567ar

Aller au sommaire du numéro

\section{Éditeur(s)}

Association canadienne de traductologie

ISSN

0835-8443 (imprimé)

1708-2188 (numérique)

Découvrir la revue

Citer ce compte rendu

Bouchard, C. (2003). Compte rendu de [Dupuis, Hector, Romain Légaré et Michel Therrien, Dictionnaire des synonymes et des antonymes, Éditions Fides, Montréal, 2003, 720p.] TTR, 16(1), 252-253. https://doi.org/10.7202/008567ar d'utilisation que vous pouvez consulter en ligne.

https://apropos.erudit.org/fr/usagers/politique-dutilisation/ 
Cela dit, on apprend mille choses dans ce livre qui vise un public assez large et qui devrait être utile pour tous ceux qui s'intéressent à l'histoire du Québec, quel que soit leur domaine de spécialisation. On s'aperçoit en effet que la langue est un des fils conducteurs les plus solides pour comprendre la logique des changements qui marquent l'histoire du Québec. En cela, ce livre complète bien des ouvrages de référence comme l'Histoire du Québec contemporain (Boréal). Ajoutons qu'il est superbement illustré et que la langue y est, comme de juste, toujours soignée.

\section{Michel Biron Université McGill}

\section{Dupuis, Hector, Romain Légaré et Michel Therrien, Dictionnaire des synonymes et des antonymes, Éditions Fides, Montréal, 2003, 720p.}

Parmi les ouvrages de référence qu'on devrait posséder lorsqu'on pratique la traduction ou la rédaction, peu sont aussi utiles qu'un bon dictionnaire de synonymes. Compte tenu de notre contexte nord-américain, la publication d'un tel ouvrage conçu au Québec a donc de quoi soulever notre intérêt, car les ouvrages français de ce type ne rendent guère compte de notre vocabulaire. Il existe par ailleurs tout un éventail de possibilités pour la présentation des données lexicales que peut contenir un dictionnaire de synonymes, de même que des critères assez variés, d'un éditeur à l'autre, pour la sélection de ces données et du type d'articles.

Les Éditions Fides viennent de publier une toute nouvelle édition du Dictionnaire des synonymes et des antonymes qui présente une refonte complète et une mise à jour de cet ouvrage de référence dont la première édition, œuvre de Hector Dupuis, remonte à plus d'une quarantaine d'années et qui avait déjà fait l'objet d'une refonte réalisée en 1975 par Romain Légaré. Outre l'ajout d'un grand nombre de nouvelles entrées qui augmentent le volume d'une centaine de pages, l'auteur de la refonte de 2003, Michel Therrien, a profité de l'occasion pour perfectionner considérablement la présentation et enrichir les articles concernant chaque entrée. Ainsi, lorsqu'un mot d'entrée possède plusieurs séries de synonymes correspondant à ses diverses acceptions, les séries sont numérotées, et les séries d'antonymes qui leur correspondent sont numérotées suivant le même ordre. Plutôt que 
de donner des définitions ou de gloser les synonymes pour en faire ressortir les nuances, chaque série de synonymes présente un motclé en caractères gras italiques qui indique le sens général de la série, de telle sorte qu'il est aisé de repérer d'un coup d'œil l'ensemble des synonymes exprimant l'acception particulière qui nous intéresse et, grâce à la numérotation, les antonymes correspondants, ce qui est parfois bien utile puisqu'il arrive que le contraire négativé fournisse exactement la nuance que l'on recherche. De nombreuses précisions concernant le contexte d'utilisation, le niveau de langue, le domaine d'emploi ou l'usage géographique sont indiquées à l'aide d'abréviations entre parenthèses. Notons qu'un grand nombre de québécismes de niveau courant et clairement identifiés ont été ajoutés à cette nouvelle édition, la précédente version du dictionnaire ne contenait en effet qu'une vingtaine de « canadianismes de bon aloi ».

L'ensemble des conventions, peu nombreuses, est présenté avec une grande clarté sur deux pages au début de l'ouvrage. Grâce à ce programme à la fois simple, précis et clair, et à une mise en page nette et bien lisible où on a évité à bon escient de multiplier les types de caractères ou les symboles, la consultation du Dictionnaire des synonymes et des antonymes est aisée et agréable. Ajoutons que la solide reliure et la couverture cartonnée devraient résister à des années d'usage, une qualité essentielle pour un ouvrage de référence.

En somme, il s'agit-là d'un instrument de travail bien conçu et fort utile pour les écrivains, rédacteurs, traducteurs, réviseurs et de manière générale, pour tous ceux qui ont régulièrement à écrire.

Chantal Bouchard Université McGill 